\title{
CARDIAC DISEASE IN CHILDHOOD, WITH SPECIAL REFERENCE TO PROGNOSIS *
}

\author{
CHARLES HUNTER DUNN, M.D. \\ BOSTON
}

In $1906 \mathrm{I}$ reported the results of an investigation of certain clinical aspects of rheumatic fever in childhood, which was based on a series of 300 consecutive patients admitted to the wards of the Children's Hospital. Of these 300 patients, 209 were discharged from the hospital with signs of organic valvular disease of the heart. The after history of these cases appeared to me to be a point of great interest, with a practical value bearing directly on the question of prognosis, and on certain aspects of treatment. I determined at the time to keep in communication as far as possible, with all of these discharged patients, and at the same time the scope of the investigation was extended beyond the limit of cardiac disease of rheumatic origin, by including all other patients of cardiac disease admitted to the hospital during the period from which the original series was taken. Cases of patients who subsequently died from intercurrent infection were thrown out.

One of the features of greatest interest in such an investigation is the amount of disability carried into adult life, or at least young adult life, by these patients. It was therefore necessary to follow the afterhistory of these patients for a period sufficiently long to afford evidence on this point. 'The period in which the cardiac patients of this series were admitted to the hospital was that of a number of years previous to the summer of 1903. I resolved to follow the cases discharged from the hospital until all of them should have entered at least young adult life, taking the age of 14 years as the termination of childhood. All of these patients have now passed this limit, the youngest survivor having reached the age of 15 years.

\section{ETIOLOGY}

The series of cases included in this investigation numbers 304 cases of cardiac disease. It throws a certain amount of light on the relative frequency of oceurrence of the various etiologic factors.

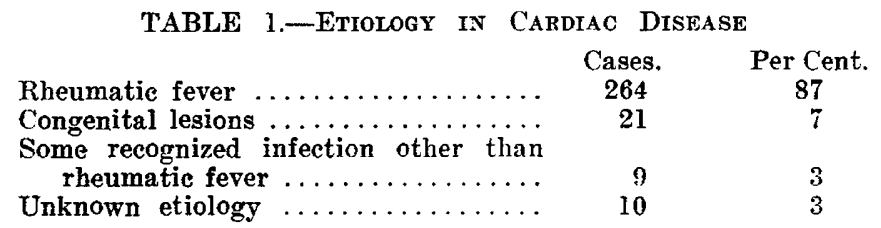

* Submitted for publication May, 1913. 
In compiling this table, in the absence of any laboratory or bacteriologic proof of the existence of rheumatic fever as a specific disease entity, the following clinical evidence of the rheumatic origin of the lesions was employed. All cases which had either during the attack in the hospital, or in previous, or in subsequent attacks, shown symptoms of arthritis or of chorea were classified as manifestations of rheumatic fever. In addition, certain cases of acute endocarditis and pericarditis with fever, in which the clinical picture was precisely similar to other cases in which arthritis did occur, and in which there was no evidence of any other form of infection, were also included. On this basis of classification, no case of acute febrile cardiac disease fell under the heading of unknown etiology, every such case being either due to rheumatic fever, or to some other recognized infection. The cases of unknown etiology were all cases of chronic endocarditis.

The table shows the overwhelming preponderance of rheumatic fever as a cause of cardiac disease.

\section{THE CARDIAC CASES DUE TO RHEUMATIC FEVER}

Acute and Chronic Cases.-Patients entering a hospital with cardiac disease, are admitted either on account of symptoms connected with the disease from which they are suffering, or the cardiac lesion is discovered accidentally in connection with the investigation of their illness. In the 264 rheumatic cases, all patients were admitted because of symptoms of this disease, either arthritic or cardiac. Arthritic symptoms in rheumatic fever, under the present most generally accepted theories of the etiology of this disease, may always be regarded as evidence of the existence at the time, of an active infection. Cardiac symptoms, on the other hand, may occur from two causes. They may be due to the existence at the time of an active infection localized in the heart, or they may be due to the so-called broken compensation, caused by calling on a damaged heart to perform a work which is too great for its power. All the cases in the series which had, during their stay in the hospital, symptoms of arthritis, or in which the cardiac symptoms were accompanied by the regular febrile course characteristic of an acute infection, are classified as acute infections. Those cases in which the cardiac symptoms were unaccompanied by fever, and in which there was no history of an immediately preceding febrile attack, are classified as chronic endocarditis.

The relative proportions of acute and chronic cases in the series are shown in Table 2.

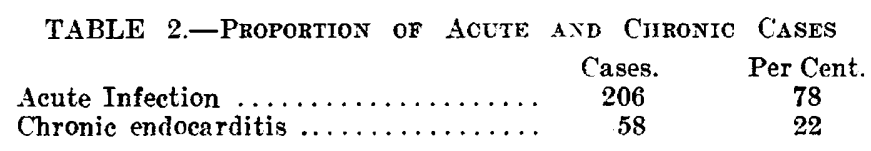


It appears, therefore, that in childhood, the causes which lead a patient to seek hospital treatment, are more often the symptoms produced by the acute infection with rheumatic fever, than those produced by its after-effects on the heart.

Relation of Cardiac Symptoms to Infection.-In my former paper I expressed the view that in childhood, as distinguished from adult life, cardiac symptoms are more often due to an actual present infection localized in the heart, than to the overstraining of a permanently damaged heart. I believe that the converse of this view is also true. In adult life, the development of an acute endocarditis in the course of an attack of rheumatic fever, is usually described as an insidious process, manifesting itself by the development of an endocardial murmur. In childhood the occurrence of an acute endocarditis is usually accompanied by actual symptoms referable to the heart. The set of symptoms called cardiac, such as precordial pain, palpitation, cough, dyspnea, orthopnea and eventually enlarged liver and edema, are in adults almost always attributed to overstrain. In childhood, this train of cardiac symptoms undoubtedly may be due to this cause, but in the majority of cases it is due to the existence of an actual present cardiac infection. Of course the symptoms are due to an inability of the heart properly to perform its work, but the cause of this inability is to be found in a fresh cardiac infection. The incompetence of the heart is not dependent on the actual amount of valvular insufficiency or stenosis present, for it bears little or no relation to the lesion. It is probably due to the acute myocarditis; which is known to be a frequent, if not an almost invariable, accompaniment of acute rheumatic endocarditis. The accompanying tables give evidence of the relation of cardiac symptoms to the presence of an actual infection :

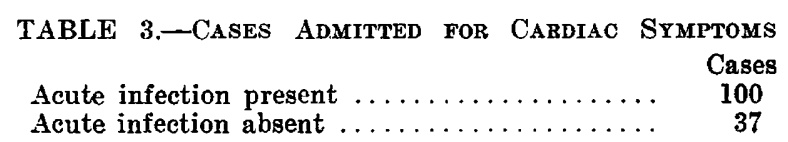

This consideration is of interest in reference both to prognosis and treatment, for it is recognized that the cause of death in the majority of these cases is cardiac failure. If the symptoms leading to such failure are most frequently due to infection, or reinfection, and not to overstrain, this fact has an obvious practical bearing.

In any event I wish to deprecate the use of the term broken compensation as applied to the train of cardiac symptoms described under this name when they occur in childhood. Apparently in only a relatively small number of cases is this term, and the theory on which it is based, applicable to the clinical picture as seen in childhood. In the majority of cases, the cause is not overexertion, but fresh infection. The term 
cardiac insufficiency is far preferable to broken compensation, as it is equally applicable to both causes.

The Cardiac Lesions.-The relative frequency of occurrence of the various cardiac lesions is of interest in connection with the subsequent history of these cases. The relation of the form of lesion to prognosis is still a more or less unsettled question in the cardiac disease of childhood. The cases, classified on the basis of the lesions shown during their stay in the hospital, were as shown in Table 4 . In only two cases was pericarditis found without any evidence of a valvular lesion in addition. The fifty-eight cases of pericarditis are classified both as pericarditis and according to the endocardial lesion present, and fifty-six of them are counted twice.

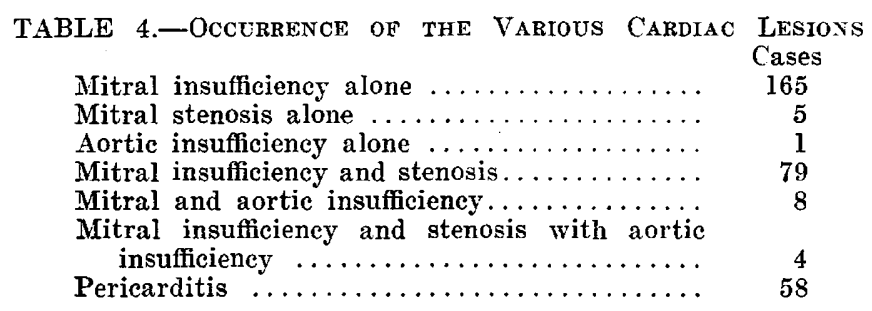

Liability in Childhood to Recurrent Attacks of Rheumatic Fever.The liability in any child attacked by rheumatic fever to recurrent attacks is probably the most important factor in all considerations of the prognosis of the disease. In my first paper the liability to recurrence could only be judged on the basis of the history of attacks of rheumatic fever previous to the one under treatment in the hospital. At present, additional evidence is available, from the following of the subsequent history of the patients throughout the remainder of their childhood, and into adult life, through a period of at least nine and one-half years.

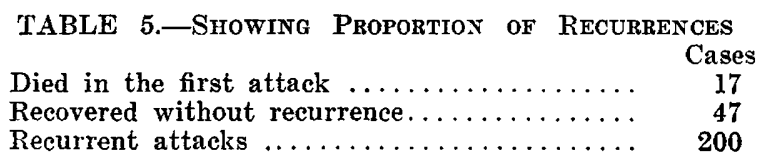

It will be seen that more than one attack of the rheumatic fever infection occurred in 76 per cent. of the cases. Only 17 per cent. showed no recurrence, as in the remaining 7 per cent. no conclusions could be drawn.

Mortality.-The complete investigation of this series of cases seems to me to be of considerable value in connection with the question of the mortality in children with hearts attacked by the rheumatic fever infection. The mortality of the cases in the hospital affords a basis for estimating the liability to death in any one attack of the disease. This is shown in Table 6 . 
Table 6.-Immiate Mortatity of Rheumatic Cardiac Disease

Died in hospital $\ldots \ldots \ldots \ldots \ldots \ldots \ldots \ldots \ldots \ldots$

Discharged, relieved $\ldots \ldots \ldots \ldots \ldots \ldots \ldots \ldots \ldots .209$

This gives an immediate mortality of 20 per cent. The immediate mortality apparently varies with the character of the process present, as shown in Table \%.

Table 7.-Immediate Mortality in Reration to the Cardiac lesiox

Acute endocarditis . . . .27 out of 148 cases or 12 per cent.

Acute pericarditis* .....18 out of 58 cases or 31 per cent.

Chronic endocarditis .....10 out of 58 cases or 17 per cent.

* In all but two of these cases acute endocarditis was probably also present.

Table 7 also shows how much more frequently death occurs as a resuli of an acute infection with rheumatic fever, than as a result of the overstraining of a chronically damaged heart. In forty-five of the fifty-five fatal cases the patient died in the course of an attack of acute infection, so that the mortality from this cause is 82 per cent. of the total.

The subsequent mortality of the cases in this series represents the chances against a child who has recovered from the attack of rheumatic fever in which he is first seen, but who is left with an organic cardiac lesion. 'The figures are shown in Table 8.

TaBle 8.-Subsequent Mortality of Rheumatic Cardiac Cases Discharged FROM THE HOSPITAL

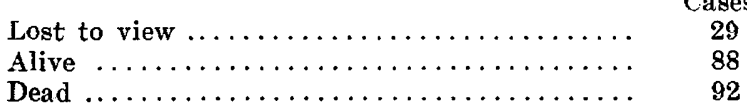

This gives a subsequent mortality of at least 23 per cent. of all cases, and if, as is fairer, the cases lost to view are thrown out, the mortality of these cases is 51 per cent.

The final mortality is that of all cases whether death occurred in the original investigation or after the discharge of the patient from the hospital. It represents the general mortality of rheumatic cardiac disease for ten years, and the chances, so to speak, in the next ten years, against any child attacked by rheumatic fever with cardiac involvement. It is shown in Table 9.

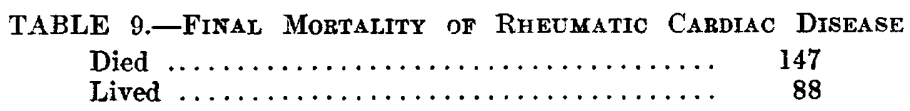

This gives the final mortality as 63 per cent. This of course represents, not the mortality of rheumatic fever, but only of the cases of rheumatic fever in which the heart is affected. During the period from which the figures of this paper were taken, only 17 patients with rheu- 
matic fever were discharged from the hospital without evidence of a cardiac lesion. Of these $1 \%$ patients, 5 remained well, 5 were lost to view, and 7 had recurrence of acute infection with cardiac involvement. Of these latter, 3 died. Including the 12 cases of rheumatic fever which could be followed, the final mortality of rheumatic fever in childhood is 60 per cent.

Relation of Mortality to Age and to Acute Infection.-This relation is one of the most interesting results of the present investigation. The basis on which the conclusions are drawn is shown in Table 10.

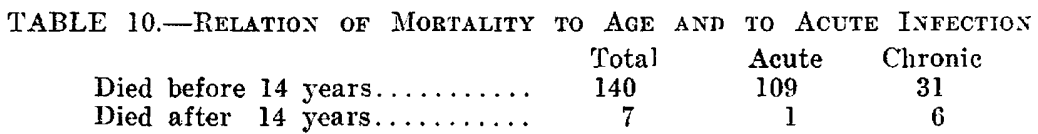

In this table the cases placed under acute are those in which the patient died in an acute febrile attack, while those placed under chronic died from cardiac failure apparently following the weakening of the heart from overstrain. It appears from the table that a great majority of the patients died as the result of an attack of rheumatic fever, and not as a result of chronic endocarditis. Even among the ninety-two patients who died after their discharge from the hospital, the majority, sixty-five, died in, or shortly after, an acute febrile attack. Such an attack was the cause of the cardiac symptoms producing death. Therefore not only the figures showing the immediate, but also those showing the final, mortality of rheumatic cardiac disease, point to infection as the chief cause of death.

But when the age at which death occurred is considered, the results are of still greater interest. The average age of all the patients at the time of the original investigation was 9 years. 'Therefore, ten years having elapsed since, at least an equal amount of childhood and young adult life has been lived by these patients. As more than ten years have elapsed since the majority of the original records were taken, more years have been spent over than under 14 years. Nevertheless, the table shows that the great majority of the patients in which a fatal ending occurred, died while still in childhood. Of the ninety-five patients who passed their fourteenth year, only seven have died, while 140 patients died in childhood. These results surely suggest that rheumatic fever is a vastly more serious and fatal disease in childhood.

The significance of the particular valvular lesions present during the patients' stay in the hospital in connection with the mortality of rheumatic cardiac disease does not appear to be great. The study of the valvular lesions in the fatal cases is not conclusive, because of the great preponderance of mitral insufficiency. The only result which is at all 
suggestive, is that no patient in whom the aortic valve was affected is now alive.

I believe there are three causes of the very great mortality of rheumatic fever in childhood. First, the greater liability of children to be attacked with rheumatic fever; second, the greater liability of children to recurrence; third, the greater liability of children to cardiac involvement. I cannot give figures in support of all these statements, but the facts are well known to most pediatrists. Table 10 shows that only one patient died after 14 years of age as the result of recurrent acute infection, the other six dying from chronic endocarditis. But 109 patients died under 14 years of age as the result of an acute infection.

We can only conclude that the chief danger in children with rheumatic cardiac ciisease is in childhood. The danger of death even from chronic endocarditis is greater, as thirty-one out of 140 patients died of chronic endocarditis in childhood, whereas six out of ninety-five patients died from this cause in young adult life. The chief cause of the danger during childhood, however, is the liability of children to recurrent attacks, in which an acute infectious process localized in the heart is the cause of the cardiac insufficiency leading to a fatal ending.

The Disability Following Rheumatic Cardiac Disease Acquired in Childhood.-This is a question of the greatest importance. The survival of eighty-eight patients now under observation, all of whom have entered young adult life, gives a basis for conclusion. The amount of disability is shown in Table 11.

Table 11.-Disability in Cardiac Disease acquired in Childhood Observed IN Young Adult Life

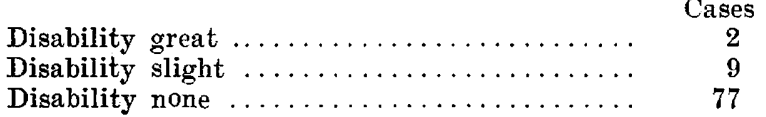

The patients with great disability are unable to work or to lead normal lives. Both of them have been in an adult hospital with broken cardiac compensation. Cardiac symptoms are brought on by comparatively slight exertion. The patients with slight disability have dyspnea on exertion, and some have occasional cough and slight edema. None of them has had an attack of severe broken compensation. They are able to work at sedentary occupations. Seventy-seven patients have no disability. Most of them work, and all of them are apparently leading peifectly normal lives, having had no cardiac symptoms since their childhood. My last set of reports contain many references to activity in dancing on the part of the girls, and in basketball and baseball on the part of the boys. One of the latter asked my permission to enter the twenty-five-mile marathon race. All of these eighty-eight patients still 
had their cardiac murmurs when I last saw them. The relation of the amount of disability to the particular valvular lesion is shown in Table 12 .

TABLE 12.-Relation of Disability to the Valvular Lesion

Great disability ....2 2 patients have mitral insufficiency

Slight disability .... 8 patients have mitral insufficiency

1 patient has mitral insufficiency and stenosis

No disability .....56 patients have mitral insufficiency

19 patients have mitral insufficiency and stenosis

2 patients have mitral stenosis

When the relative occurrence of the various lesions is taken into account, this table shows no relation between the amount of disability and the character of the valvular deformity.

The relation of the amount of disability to the age at which the patient had his first attack of rheumatic fever is shown in Table 13.

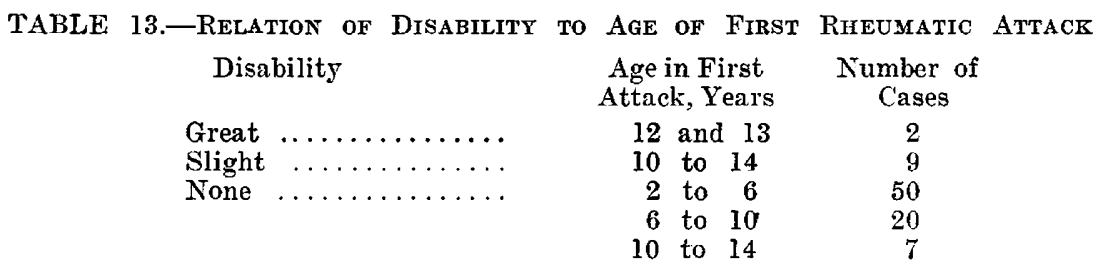

This table shows that all the patients with disability were attacked when past the age of 10 years, whereas in the cases without disability, the majority were first attacked in early childhood. It suggests that the earlier in childhood a cardiac lesion is acquired, the better will be the result as regards the ability to lead a normal adult life.

It seems to me that the general amount of disability seen in rheumatic cardiac disease acquired in childhood is remarkably small. I believe it to be very much less than the disability following rheumatic endocarditis acquired in adult life. Unfortunately, I cannot give figures bearing on this point, as, while rheumatic fever is fairly common in adults, it attacks the joints alone with such greater frequency than in childhood, that cases in which endocarditis develops for the first time in adult life are comparatively rare. I have not had time to accumulate a sufficient number of such cases to afford a basis for comparison. I believe, however, that such a comparison would show a much less disability in cardiac disease acquired in childhood. My recollection of my work in an adult out-patient department, and in a general hospital, is that the majority of patients suffering from broken compensation or serious disability from chronic endocarditis, could trace the origin of their lesion, if at all, to some attack of rheumatic fever occurring since they had entered adult life. On the other hand, in every adult outpatient department are seen many patients who are seeking medical aid 
on account of some affection other than cardiac, in whom, in the course of routine examination, some valvular lesion of the heart is found. In a large number of cases these patients declare that they have never suffered from cardiac symptoms, and have no recollection of any attack of rheumatic fever. I believe that in very many of such patients, the cardiac lesion represents the sequela of an attack of rheumatic fever which occurred in childhood, at an age when the arthritic manifestations are often or so much less pronounced, that they are not remembered. These patients, leading normal lives, yet with every evidence of organic cardiac disease, are common. They correspond to the seventy-seven cases in my series in which no cardiac disability persisted.

A possible explanation of this lessened disability in cardiac diseast: acquired in childhood, is suggested by the inferences from Table 13. The disability appears to be less, the earlier in childhood the lesion is acquired. In many of the permanent lesions acquired in childhood, the power of adaptation between. the damaged organ and the work required of it is greater, and children show a marvelous power of adapting themselves to the requirements of life. In the case of cardiac disease, I believe the so-called compensation of the damaged heart is more than a mere mechanical hypertrophy. I believe that when the lesion occurs during childhood, that is, during the period of active growth, a mutual adaptation between the heart and the child gradually occurs, which is far more perfect than can occur after the period of growth is passed. The child grows up to fit its heart, and the heart develops to fit the child. Only in this way can I explain the facts suggested by this paper. The earlier in life the damage to the heart, the longer is the period of growth during which this adaptation can occur.

Inferences as to the Treatment of Cardiac Disease of Rheumatic Origin.--The outcome of the patients discharged from the hospital is suggestive as regards treatment. Treatment in the chronic endocarditis of childhood resolves itself largely into the question of prophylaxis. If the sole danger to be apprehended were the rupture of campensation by overstrain, prophylaxis would consist simply in the guarding of children with damaged heart valves from overexertion. But we have seen that the chief danger lies in the liability to a recurrent attack of the infection. We know so little of the exact nature of the infecting organism in rheumatic fever, and of its channels of invasion, that we can accomplish comparatively little in preventing recurrent attacks, beside the most general hygiene measures. The evidence in favor of the tonsil as a frequent route of invasion is so great, that, in view of the fatality of the disease, $I$ believe we are justified in advocating the removal of the tonsils in every patient who has had one attack. If a patient who, in addition to having had an attack of rheumatic fever, is subject to tonsillitis, removal 
of the tonsils is positively indicated. The frequency and severity of rheumatic fever appears to vary somewhat with climate and locality. Whenever it is possible, I believe we should lessen the chances of reinfection by removing the patients to a place where rheumatic fever is uncommon.

The most important question is how far we should go in guarding against the breaking down of cardiac compensation by overstrain and overexertion. That children are liable to danger from this cause is a fact, as shown by the thirty-one patients in this series who died from chronic endocarditis while still within the limit of childhood. That we should endeavor to guard against dangerous overstrain goes without question. But it is questionable how far our efforts should reach in limiting the normal activities of the child. We must consider the question of lessening the disability in adult life, when the heart must be able successfully to cope with the strain of a normal active life. If such ability is due to a gradual adaptation between the child and the heart, it is very probable that too much limitation of the activities of childhood will prevent this adaptation from taking place, and will leave the child unfitted to encounter the demands of active adult life.

Most of the parents of the patients in my series were given strict directions as to the guarding of their children from overstrain, and as to the limiting of their activities. Some of them followed these directions, while the majority disregarded them entirely. Singularly enough, of those who obeyed these directions at all, the majority obeyed them with exaggeration, limiting the activities of the children to an extreme degree. The relation of the limitation of activity to the mortality from chronic endocarditis is shown in Table 14.

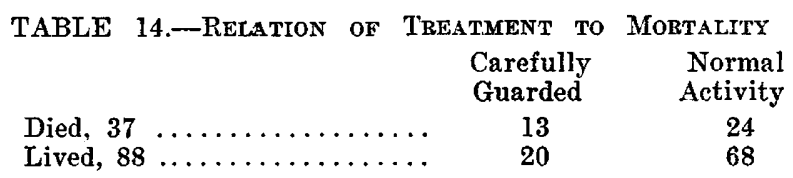

The proportion of children who lead a life of normal activity during childhood is greater in those who lived than in those who died. It does not seem from this series at least as if the guarding of children from overexertion had any notable effect on the mortality. Moreover, when the cases are analyzed as to the relation between limitation of activity and subsequent disability, it appears that in general those children did better in whom the normal activities of childhood were not limited at all.

I do not mean that we should let our cardiac patients engage in all normal activities without limit, or without supervision. I simply wish to point out the possibility of going too far toward the other extreme. I believe we should avoid putting our cardiac patients under a glass case, 
for even normal activity is preferable to extreme limitation of activity. We should remember the other aspect of the question, that of the gradual adaptation of the child to the damaged heart, which takes place during the previous period of growth. We must do all in our power to favor this process, and this can only be done by allowing the heart to accustom itself to increasing demands on its power during this period. Nothing can be more important than the proper management of these cases, for I believe that the future of the child is largely dependent on it. The most thorough supervision is essential. Beginning at an early period after the disappearance of symptoms, with passive motion against increasing resistance, we should constantly increase the amount of activity allowed, by regulated exercises, so long as we can keep within the danger limit, of which the warning signs are extreme rapidity of cardiac action, or slight dyspnea. But we should not stop when a moderate amount of activity can be indulged in without symptoms. We should continue to increase the amount of activity allowed, and if finally these children can indulge in all the activities of childhood without symptoms, I believe we should not hesitate to remove all limitations. Their chief danger during childhood lies in reinfection, not in overexertion, and the more they learn to do in childhood, the better are their prospects for a normal, active, useful adult life.

\section{CONGENITAL CARDIAC DISEASE}

The cases of congenital cardiac disease in this series are too few to permit the drawing of conclusions of any great value. There were twenty-one cases.

Open Ductus Arteriosus. - Fifteen cases were diagnosed as open ductus arteriosus. The patients had a murmur, but no enlargement of the cardiac dulness. Two of them had slight cyanosis but no thrill, and two had a palpable thrill but no cyanosis.

The final result in these cases was as follows: Two died, four were lost sight of and nine recovered. The two patients who died, were those having slight cyanosis, and who died in infancy. In one the diagnosis was confirmed at autopsy. Both of the cases in which there was a palpable thrill recovered, with disappearance of the thrill. In all of the nine cases of recovery, and in two of the patients lost sight of, the murmur eventually disappeared, but I am unable to state the exact time of its disappearance. The nine patients are now apparently well, and are between 10 and 16 years of age.

Pulmonary Stenosis. - Five cases were diagnosed as pulmonary stenosis. The patients had a murmur, cyanosis, palpable systolic thrill and enlargement of the area of cardiac dulness. Of these cases, four patients died before they were 2 years old, and one recovered. This patient is still very cyanotic, with the same signs and clubbed finger tips. 
Deficient Ventricular Septum.--In one case this diagnosis was made. There was a murmur, enlargement of the cardiac dulness, no cyanosis and no thrill. This patient, seen at 8 months, lived a year, and was then lost sight of.

It would seem that open ductus arteriosus is a comparatively favorable lesion. The most interesting feature is the disappearance of the murmur in the patients who recovered. As open ductus arteriosus is due to a failure of the closing of the ductus by normal obliterating endarteritis, I believe the disappearance of the murmur suggests that the closure is simply delayed, and may normally occur later. In these cases, without thrill, cyanosis, or enlargement, I believe that as far as the cardiac lesion is concerned, a most favorable prognosis should be given.

\section{ENDOCARDI'ISS OF OTHER ORIGIN THAN RHEUMATIC FEVER}

Beside the congenital cases, there were only nineteen cases of endocarditis in the series which could not be connected with the rheumatic fever infection. Of these, ten cases were of unknown origin. Rheumatic fever could not be excluded, but no connection with this infection was found either in their past or subsequent history. Of these cases, four patients were lost sight of, three entered adult life with signs of mitral insufficiency, but no disability, and in the remaining three the murmur disappeared and they are well.

of the nine remaining patients, two were fatal cases of purulent pericarditis following pneumonia, and two were fatal cases of malignant endocarditis following an alveolar abscess and mastoiditis, respectively. In the other five cases the cardiac lesion was attributed to scarlet fever. Two of the patients were lost sight of and three have no disability. In one of these the murmur has disappeared.

\section{CONCLUSIONS}

1. Rheumatic fever is very much the commonest cause of cardiac disease in childhood.

2. Cases with acute rheumatic infection localized in the heart are much commoner than cases suffering from chronic endocarditis.

3. Cardiac symptoms are due to two causes: first, acute infection localized in the heart; second, broken cardiac compensation. Of these two causes the first is the commoner.

4. The liability of children to recurrent attacks of acute rheumatic infection, in any of which the heart may be involved, is very great.

5. The immediate mortality of rheumatic cardiac disease is about 20 per cent.

6. The subsequent mortality of patients with endocarditis of rheumatic origin, followed for at least ten years in about 50 per cent. 
7. The final mortality of rheumatic fever followed for at least ten years is 60 per cent.

8. The mortality is seen chiefly during childhood. The mortality after young adult life is reached falls to only 7 per cent.

9. The cause of death is heart failure. The cause of the heart failure may be either acute cardiac infection or broken compensation. In childhood the former cause is far the more common. After adult life is reached the latter cause is more common.

10. The particular valvular lesion present has little or no relation either to the mortality or the amount of disability in adult life; except that aortic disease appears to be a particularly fatal lesion in childhood.

11. 'The causes of the great mortality of rheumatic fever in children are, first, their greater liability to this infection; second, their greater liability to recurrent attacks; third, their greater liability to cardiac involvement.

12. Patients who escape the dangers of childhood, and who enter adult life, are apt to show a remarkable freedom from disability. The majority of such patients can lead normal active lives.

13. The probable cause of this freedom from disability lies in the fact that the cardjac damage occurs during the period of growth, and during this period a particularly perfect adaptation can take place between the heart and the patient, which enables the heart to meet the demands made on it. This adaptation is more perfect than can be attained in the adult.

14. The earlier in life the cardiac lesion is acquired, the better is apt to be the result in adult life, as concerns ability to lead an active, normal existence; provided that the patient escapes the dangers of childhood.

15. Treatment should be directed toward favoring the adaptation of child and heart. While guarding against overstrain, we must avoid too great limiting of the normal activities of childhood.

16. In congenital cardiac disease, open ductus arteriosus is a favorable lesion.

1781 Marlboro Street. 